\title{
Incubation of anxiety: Effect on temporal generalization'
}

\author{
OTELLO DESIDERATO², JUDITH M. FOLDES AND JOAN S. GOCKLEY
}

Temporal generalization gradients were obtained, either immediately after training or after a 6-hr. delay, from Ss exposed to either aversive or innocuous reaction time signals. Smaller generalization decrements were found for Ss receiving delayed testing, and signal aversiveness did not affect the magnitude of the decrement. Instead of finding support for an "anxiety-incubation" hypothesis, it was concluded that non-emotional factors associated with elapsed time produce flattened generalization gradients.

For human Ss, the effect of delayed testing on the PGR has been to elevate, but not flatten, the generalization gradient, suggesting an increase, or "incubation," of conditioned anxiety across time (Mednick, 1957). However, several acquired-drive studies have indicated that, following classical aversive conditioning, rats show smaller generalization decrements-without elevation of the overall gradient-if time elapses between conditioning and testing (McAllister \& McAllister, 1963; Desiderato, Butler, \& Meyer, 1966).

While it is possible that the "incubation effect" may be limited to human Ss, wide procedural differences make comparison between these studies difficult. Accordingly, the present study is an additional test of the "incubation effect" at the human level, using a temporal generalization paradigm. To test whether gradient effects associated with delay necessarily involve changes in conditioned emotionality, for half the Ss, the training phase did not involve aversive stimulation. Thus, gradient effects could be attributed to an "incubation" process only in the case of Ss tested following aversive training.

\section{Method}

The Ss were 40 college women. ${ }^{3}$ Following the procedure for obtaining temporal gradients described elsewhere (Desiderato, 1964), each $S$ was trained for 25 trials to press a telegraph key in response to an auditory signal delivered via earphones once every $12 \mathrm{sec}$. Since the S's response immediately terminated the signal, signal duration was equivalent to her reaction time. Throughout the experiment, for half the Ss, the signal was white noise at an aversive level of $119 \mathrm{~dB}, \mathrm{SPL}$ (Group N); the remaining Ss heard a pure tone of $1000 \mathrm{cps}$, at $86 \mathrm{~dB}$, SPL (Group T).

Following the 25th training trial, for half the Ss in each group the generalization test commenced without interruption. Ss in the remaining subgroups were asked to return for a continuation of the experiment $6 \mathrm{hr}$. later. Every $S$ received five testblocks, each consisting of seven trials. Within each block, the following interstimulus intervals were used: $5.5,7.0,9.0,12.0,15.5$,
20.0 , and $26.0 \mathrm{sec}$. The order varied randomly from $S$ to $S$ and from block to block. After the last test trial, Ss were asked to indicate on 5-point rating scales how unpleasant they found (1) the signal to respond (noise or tone) and (2) the entire experiment.

\section{Results and Discussion}

All RT scores were transformed into reciprocals. Analysis of training trials revealed that Group $\mathrm{N}$ responded faster than Group $T, F=34.71, d f=1 / 36$, $\mathrm{p}<.001$, and that response speed increased during training, $F=38.89, \mathrm{df}=4 / 344, \mathrm{p}<.001$. For the generalization test data, separate analyses of RTs to the three intervals longer and shorter than to the 12-sec. training interval both yielded nonsignificant F-ratios.

In light of the failure to obtain falling gradients, a different measure of generalization was devised. This consisted of comparing each S's mean response speed for the last five training trials with her mean response speed to all six generalization test intervals combined. This comparison, while precluding the opportunity to see whether an incubation effect would elevate responsivity to all test intervals, including the 12-sec.training interval, nonetheless does show how large a generalization decrement occurs when testing is immediate or delayed. "Incubation" should attenuate the decrement in the Noise-Delay subgroup more than in the ToneDelay subgroup. However, if changes in gradient slopes were to result from non-emotional processes associated with time, then approximately equal decrements would be expected to occur in these two groups. Figure 1 shows a generalization decrement for each of the four

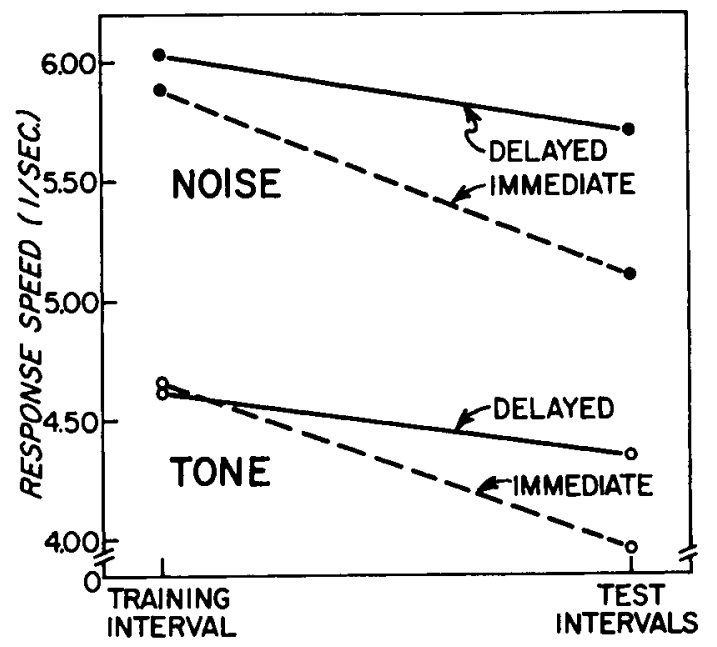

Fig. 1. Temporal generalization gradients showing speed of response of Noise and Tone groups. 
experimental subgroups. Mann-Whitney U-tests indicated that, under both the Noise and Tone conditions, the decrement was significantly smaller for Ss receiving delayed testing, ( $\mathrm{U}=107,112$, respectively, both ps $<.01$, one-tailed). Application of a U-test revealed that the difference between the mean decrements shown by the two delayed subgroups was not significant $(\mathrm{U}=$ 182).

These generalization data, while limited to gradients which include response strength to both training and generalized temporal intervals, do notprovide evidence for an "incubation effect" in humans. The possibility that the two auditory signals were not differentially aversive cannot easily account for the similar decrements in the two stimulus groups, for $U$ tests of rating scale data indicate that the Noise group found both (1) the signal and (2) the entire experiment significantly more unpleasant than did the Tone group $(z=4.42$, $p<.0001$, and $z=2.42, p<.01$, respectively).

We offer the suggestion, therefore, that the flatter gradients associated with delayed testing may be due to non-emotional processes, e.g., memory for the interval used in training, dissipation of temporal sets, etc., rather than resulting from increased anxiety. This view is compatible with generalization data obtained from animals following instrumental reward training (Perkins \& Weyant, 1958), and with the results of a recent psychophysical study with human Ss which shows that time elapsing between the presentation of constant and variable stimuli impairs discrimination of line lengths (McAllister, McAllister, \& Franchina, 1965).

\section{References}

Desiderato, $\boldsymbol{O}$. Effect of anxiety and stress on reaction time and temporal generalization. Psychol. Rep., 1964, 14, 51-58.

Desiderato, O., Butler, B., \& Meyer, C. Changes in fear generalization gradients as a function of delayed testing. J. exp. Psychol., in press.

McAllister, W. R., \& McAllister, D. E. Increase over time in the stimulus generalization of acquired fear. J. exp. Psychol., 1963, $65,576-582$.

McAllister, W. R., McAllister, D. E., \& Franchina, J. J. Dependence of equality judgments upon the temporal interval between stimulus presentations. J. exp. Psychol., 1965, 70, 602-605.

Mednick, M. T. Mediated generalization and the incubation effect as a function of manifest anxiety. J. abnorm. soc. Psychol., 1957, 55, 315-321.

Perkins, C. C., Jr., \& Weyant, R. G. The interval between training and test trials as a determiner of the slope of generalization gradients. J. comp. physiol. Psychol., 1958, 51, 596-600.

\section{Notes}

1. We are grateful to Rena Rimsky for statistical assistance and to George $T$. Woods for setting up the apparatus

2. Currently at the University of Pennsylvania.

3. This study was conducted twice, with 20 Ss each replication. Since no replication effects were found to be significant, pooled data for $40 \mathrm{Ss}$ are reported herein. 